\title{
THE PREVALENCE OF ENTEROTOXIGENIC E. COLI ISOLATED FROM THE STOOLS OF CHILDREN AGED 0- 10 YEARS WITH DIARRHEA IN MID-ANATOLIA REGION, TURKEY
}

\author{
Latife Işeri $^{1 *}$, Teoman Zafer Apan ${ }^{1}$, Altan Aksoy ${ }^{1}$, Figen Koç, Jülide Sedef Göçmen ${ }^{1}$, Diba Nuristani \\ ${ }^{1}$ Kırıkkale University, Department of Medical Microbiology, Kırıkkale, Türkiye. ${ }^{2}$ Child diseases hospital, Microbiology \\ laboratory, Kırıkkale, Türkiye
}

Submitted: November 03, 2009; Returned to authors for corrections: February 03, 2010; Approved: November 04, 2010.

\begin{abstract}
The stool samples from 245 patients with diarrhea were tested for heat labile toxin (LT) and heat stable toxins (ST) by passive latex agglutination and enzyme immunoassay methods respectively. Twelve (4.9\%) enterotoxigenic E. Coli ETEC strains were isolated. Five strains (2\%) expressed ST, and 7 (2.8\%) expressed
\end{abstract} LT.

Key words: Enterotoxigenic E. coli, Traveler's diarrhea, Infectious Diarrhea, labile toxin, stable toxin

Enterotoxigenic Escherichia coli (ETEC) is an important cause of acute diarrhea in childhood. Generally affected populations are newborns, young children, and susceptible tourists ETEC strains are particularly common in tropical regions. In developing countries, children suffer from diarrhea attacks 7-8 times a year and ETEC strains are responsible for one of each 3 attacks $(5,7,13)$. In developed countries, on the other hand, food and water-borne epidemics have been reported. However, the main problem for such countries is the diarrhea acquired by tourists while they are traveling in developing countries(6). ETEC has an important place among the etiologies for traveler's diarrhea.

To diagnose the presence of ETEC, heat labile toxin and/or heat stable toxin producing $E$. coli must be detected in the stool. From various parts of the world, ETEC has been reported to be the main etiological agent of diarrhea among children of $0-5$ years of age at $1.86 \%$ - $20.7 \%(1,10,16,15)$.
The main goal of this study was to investigate whether ETEC is an important factor in diarrhea in children and to know its prevalence in our region.

This study was conducted at the microbiology laboratory of Kırıkkale University Hospital. Kirikkale is a city in MidAnatolia region with a population of 280000 and land area of $4.630 \mathrm{~km}$. Life in the city is mostly urban. The samples were collected from children of 0-10 years of age who applied with complaints of diarrhea to Children's Diseases Hospital in the region and the Hospital of Kirikkale University Medical School.

Two hundred and forty-five diarrhea and 30 helthy stool samples were collected. Total 275 stool samples were inoculated onto eosin methylene blue agar plate (EMP). They were incubated at $37{ }^{\circ} \mathrm{C}$ overnight. Only one strain identified as E. coli from the stool sample of each patient was included in the evaluations. All the strains included in the study were

*Corresponding Author. Mailing address: Kırıkkale Üniversitesi, Tıp Fakültesi Hastanesi Mikrobiyoloji Anabilim Dalı. Kırıkale, Türkiye.; E-mail: liseri2000@yahoo.com 
stored at $-80{ }^{\circ} \mathrm{C}$. The strains were defrosted at 96-test groups, and tested for the production of heat labile and/or heat stable toxins.

Two-three colonies from pure cultures were put into tryptic soy broth and incubated at $37{ }^{\circ} \mathrm{C}$ for overnight. Next day, they were centrifuged at $900 \mathrm{~g}$ for 30 minutes. The supernatant was investigated for the presence of ST by Coli ST EIA test kit (Denka Seiken Co. Ltd. Tokyo-Japan). The sensitivity of this test, according to the values given by the manufacturing company, is $10-20 \mathrm{ng} / \mathrm{ml}$ and highly specific for E. coli ST+. LT was determined using VET-RPLA passive latex agglutination test kit (Oxoid. Oxoid Limited, Basingstoke Hampshire RG24 8PW, UK). Its sensitivity is $2 \mathrm{ng} / \mathrm{ml}$. E. coli strains were put into Mundell's culture medium and incubated at $37{ }^{\circ} \mathrm{C}$ overnight; the cultured Mundell's medium was reincubated at $37{ }^{\circ} \mathrm{C}$ for 4 hours after adding $10.000 \mathrm{u} / \mathrm{ml}$ polymyxin $\mathrm{B}$ as it is suggested in the kit procedure. The cultures were centrifuged at $4{ }^{\circ} \mathrm{C}, 900 \mathrm{~g}$ for 20 minutes, and supernatant was worked. Results were obtained by visual agglutination on black background.

The antibiotic resistance of 14 ETEC strains and 53 non ETEC strains, 28 of which were isolated from the diarrhea stools and 25 of which were isolated from healthy stools was studied by disk diffusion method.

The results were evaluated with chi square test in SPSS 10,0 programme.

The following antimicrobial disks were used: ampicillin $(10 \mu \mathrm{g})(\mathrm{AMP})$, amoxicillin clavulanic acid (30 $\mu \mathrm{g})(\mathrm{AMC})$, gentamycine $(10 \mu \mathrm{g})(\mathrm{GN})$, ciprofloxacin $(5 \mu \mathrm{g})(\mathrm{CIP})$, trimethoprim-sulfamethoxazole $(25 \mu \mathrm{g})(\mathrm{SXT})$ (Oxoid, Oxoid Limited Basingstoke, Hampshire, England). E. coli ATCC 25922 was used as a reference strain for quality control. The results were interpreted according to criteria of the National Committee for Clinical Laboratory Standards (11)

Of 245 diarrhea stool samples, 12 (4.9\%) ETEC strains were isolated. Of these, 5 (2\%) were ST and 7 (2.8\%) were LT. Among the E. coli strains isolated from 30 healthy stools, $2(6.7 \%)$ ST were isolated. Statistical significance difference between healthy stools and diarrhea stool samples was not considered $(\mathrm{P}>0.05)$.

The antibiogram results of $E$. coli strains isolated from the stool samples of children of 0-10 years of age have been provided in Table 1.

Table 1. Antibiotic resistance of ETEC and E. coli isolated from stool samples of diarrheic and non-diarrheic children (0-10 yearsold).

\begin{tabular}{lllllll}
\hline strain & $\begin{array}{l}\text { CIP } \\
\boldsymbol{\%}(\mathbf{n})\end{array}$ & $\begin{array}{l}\text { AMC } \\
\boldsymbol{\%}(\mathbf{n})\end{array}$ & $\begin{array}{l}\text { AMP } \\
\boldsymbol{\%}(\mathbf{n})\end{array}$ & $\begin{array}{l}\text { SXT } \\
\boldsymbol{\%}(\mathbf{n})\end{array}$ & $\begin{array}{l}\text { GN } \\
\boldsymbol{\%}(\mathbf{n})\end{array}$ & $\begin{array}{l}\text { Multiple } \\
\text { resistant \%(n) }\end{array}$ \\
\hline ETEC (14) & $21(3)$ & $29(4)$ & $36(5)$ & $21(5)$ & $7(1)$ & $29(4)$ \\
Diarrheic strains (28) & $11(3)$ & $64(18)$ & $75(21)$ & $39(11)$ & $11(3)$ & $68(19)$ \\
Healthy strains (25) & $0(0)$ & $56(14)$ & $60(15)$ & $20(5)$ & $8(2)$ & $56(14)$ \\
Total (67) & $9(6)$ & $54(36)$ & $61(41)$ & $28(19)$ & $9(6)$ & $55(37)$ \\
\hline n: the number of resistant strains, \%:resistance rate, CiP: ciprofloxacin, AMP: ampicillin, & AMC: amoxicillin clavulanic acid, SXT:
\end{tabular}

trimethoprim-sulfamethoxazole, GN: gentamycine

The resistance rates of ETEC strains to antibiotics were $21 \%$ for CIP, $29 \%$ for AMC, $36 \%$ for AMP, $21 \%$ for SXT, and $7 \%$ for GN. In the isolates from diarrhea stools, these rates were $11 \%, 64 \%, 75 \%, 39 \%$, and $11 \%$ for CIP, AMC, AMP,
SXT, and GN, respectively. In the isolates from the samples of healthy children, the rates of resistance to CIP, AMC, AMP, SXT, and GN were $0 \%, 56 \%, 60 \%, 20 \%$, and $8 \%$ respectively (Table1). No ESBL growth was observed in any of the strains 
including all the ETEC.

Statistical significance difference between resistance prevalences to antibiotics of ETEC and non ETEC strains was not considered $(\mathrm{P}>0.05$ for all analyses).

Although not statistically significant, except with CIP, the resistance rates of ETEC to all the other antibiotics were lower than those of non ETEC strains. ETEC were most susceptible to gentamycine (susceptibility rate: 93\%). The resistance rates to AMP (36\%) and AMC (29\%) were significantly high. The strains resistant to more than 2 antibiotics were considered to have multiple resistance. Multiple resistance rates were also high. Among all the strains studied with antibiogram, the strains that were resistant to GN were also resistant to AMP, AMC, and SXT, while they were susceptible to CIP. Nevertheless, considering all the strains together, ETEC strains were the most CIP resistant group of strains.

ETEC is an important etiological agent of diarrhea in developing countries. Reports from Bangladesh, Mexico, Peru, Egypt, Argentina, India, Nicaragua, and Tunisia indicate an ETEC prevalence rate of $18-38 \%$ in symptomatic children. These rates are much lower in reports from Vietnam and Brazil $(4 \%, 3.7 \%)(14,2,8,3)$. In a study conducted in our country in 2005, Ozerol et al determined a $10 \%$ prevalence rate for ETEC in children (0-5 years) with diarrhea (12). In Kirıkkale, we determined a prevalence rate of $4.9 \%$ in symptomatic children of 0-10 years of age. This rate is similar to the rates determined in Vietnam and Brazil $(8,3)$. However, it is lower than the rate reported by Ozerol et al. In the study by Ozerol et al, an age group of 0-18 years was studied and 69 samples from 0-5 years old group and 39 samples from 6-18 years (total= 108 samples) were evaluated. In that study, 7 ETEC were isolated. The prevalence of ETEC was $10 \%$ for 0-5 year-old group, while no ETEC were isolated from 5-18 year-old group. If the rate for 010 year-old group had been calculated, the ETEC prevalence rate would probably have been under $10 \%$ (12). Evaluation of the results of the study by Ozeral et al and our study shows that the prevalence rate of ETEC in our country is not high.
While LT expression was more common in the strains isolated in Mexico, Peru, Argentina, and India, ST expression was more common in the strains isolated in Bangladesh, Egypt, and Nicaragua. In these countries, the rate for simultaneous expression of LT and ST varied between $7 \%$ and $49 \%$ (14). In the study from our country by Ozerol et al, the rate of ST expression in the ETEC strains isolated from children aged 0-5 years with diarrhea was $28 \%$, while for LT expression, the rate was $71 \%$ for the same group of children (12). In our study, $41.8 \%$ of the ETEC strains isolated from children with diarrhea expressed ST, whereas $58.3 \%$ expressed LT. The ETEC strains isolated in our region express LT more frequently as the strains isolated in Mexico, Peru, Argentina, and India. Furthermore, to date, no ETEC strains that express both toxins have been detected in our region. The higher prevalence of ETEC strains that express LT than those expressing ST is an important finding because the LT expressing ETEC strains are considered to be less pathogenic than those expressing ST. The colonization factors (CF) in LT expressing ETEC are fewer than in ST expressing ETEC and frequently, they are accompanied by mild infections. The ETEC strains that express ST or LT/ST simultaneously are often isolated from severe diarrhea infections (14). In our region, the low prevalence of ETEC and among the ETEC isolated, the high prevalence rate of less pathogenic, LT expressing ETEC strains is a positive finding with regard to child health and tourism.

Although no LT expressing ETEC was isolated from the 30 -sample control group, in $2(6.6 \%)$ stools, ST expressing ETEC was isolated, which is a significant finding. The coexistence of ST expressing ETEC with colonization factors is more prevalent. Therefore, ST expressing ETEC are more likely to colonize and dwell in the bowels for a long time. In conclusion, although these children are not ill at the moment, they may pose a risk for contagion. In fact, these colonizations might be responsible for repeated ETEC attacks over a yearperiod in developing countries.

In diarrhea caused by ETEC, fluid replacement therapy is 
usually effective. However, in persistent cases, antibiotic treatment may be required. Although in the years when ETEC strains were first isolated, they were found to be susceptible to antibiotics, in time they have acquired resistance to the most commonly used antibiotics. CIP was a safe drug for prophylaxis against travel related diarrhea $(4,14)$. The resistance rates of ETEC strains to drugs have increased in the last decade. In a study from Kuwait, the resistance rates of ETEC strains were found to be $66,7 \%$ for AMP, $11,1 \%$ for GN, 11,1 for CIP. The resistance rates reported from Mozambique are $61 \%$ for SXT and $46 \%$ for AMP, $8 \%$ for AMC. In an earlier study conducted in Malatya city of Turkey, the resistance rates were reported to be $54.4 \%$ for AMP and $36 \%$ for $\operatorname{SXT}(1,9,12)$. In our study, drugs of common use were tested. The results indicated that the resistance rates of ETEC strains to antibiotics were lower than the resistance rates of non-ETEC E. coli strains. On the other hand, except with GN, the resistance rate to the other four antibiotics evaluated (CIP, AMC, AMP, SXT) and multiple antibiotic resistance rate were found to be over $20 \%$. This suggests the onset of problems in ETEC associated diarrhea requiring treatment. Although the low resistance to $\mathrm{GN}$ is an advantage, the strains that are resistant to GN are also resistant to the other antibiotics constitute another difficulty in the treatment.

In this study, a low ETEC prevalence was found. The majority of the isolated strains $(58.3 \%)$ were less pathogenic, LT expressing ETEC strains. These are positive findings with regard to child health and tourism. Nevertheless, high rates of resistance to antibiotics may lead to problems in the cases requiring antibiotic treatment.

\section{ACKNOWLEDGEMENT}

This study is supported by Scientific Research Projects Unit of Kırıkkale University

\section{REFERENCES}

1. Albert, M.J.; Rotimi, V.O.; Dhar, R.; Silpikurian, S.; Pacsa, A.S.; Molla,
A.M.; Szucs, G. (2009). Diarrhoeagenic Escherichia coli are not a significant cause of diarrhoea in hospitalised children in Kuwait. BMC Microbiol. 30, 9:62.68

2. Al-Gallas, N.; Bahri, O.; Bouratbeen, A.; Ben Haasen, A.; Ben Aissa, R. (2007). Etiology of Acute Diarrhea in Children and Adults in Tunis, Tunisia, with Emphasis on Diarrheagenic Escherichia coli: Prevalence, Phenotyping, and Molecular Epidemiology. Am J Trop Med Hyg., 77(3), 571-582.

3. Bueris, V.; Sircili, M.P.; Taddei, C.R.; dos Santos, M.F.; Franzolin, M.R.; Martinez, M.B.; Ferrer, S.R.; Barreto, M.L.; Trabulsi, L.R. (2007). Detection of diarrheagenic Escherichia coli from children with and without diarrhea in Salvador, Bahia, Brazil. Mem Inst Oswaldo Cruz. 102(7), 839-844.

4. Diemert, D.J. (2006). Prevention and self-treatment of traveler's diarrhea. Clin Microbiol Rev. 19(3), 583-594.

5. Estrada-Garcia, T.; Lopez-Saucedo, C.; Thompson-Bonilla, R.; Abonce, M.; Lopez-Hernandez, D.; Santos, J.I.; Rosado, J.L.; DuPont, H.L.; Long, K.Z. (2009). Association of Diarrheagenic Escherichia coli Pathotypes with Infection and Diarrhea among Mexican Children and Association of Atypical Enteropathogenic E. coli with Acute Diarrhea. J Clin Microbiol. 47(1), 93-98.

6. Goldsmid, J.M.; Leggat, P.A. (2007). The returned traveller with diarrhoea. Aust Fam Physician. 36(5), 322-327.

7. Harris, A.M.; Chowdhury, F.; Begum, Y.A.; Khan, A.I.; Faruque, A.S.; Svennerholm, A.M.; Harris, J.B.; Ryan, E.T.; Cravioto, A.; Calderwood, S.B.; Qadri, F. (2008). Shifting prevalence of major diarrheal pathogens in patients seeking hospital care during floods in 1998, 2004, and 2007 in Dhaka, Bangladesh. Am J Trop Med Hyg. 79(5), 708-714.

8. Hien, B.T.; Scheutz, F.; Cam, P.D.; Serichantalergs, O.; Huong, T.T.; Thu, T.M.; Dalsgaard, A. (2008). Diarrheagenic Escherichia coli and Shigella strains isolated from children in a hospital case-control study in Hanoi, Vietnam. J Clin Microbiol. 46(3), 996-1004.

9. Mandomando, I.M.; Macete, E.V.; Ruiz, J.; Sanz, S.; Abacassamo, F.; Vallès, X.; Sacarlal, J.; Navia, M.M.; Vila, J.; Alonso, P.L.; Gascon, J. (2007). Etiology of diarrhea in children younger than 5 years of age admitted in a rural hospital of southern Mozambique. Am J Trop Med Hyg. 76(3), 522-527.

10. Moyo, S.J.; Maselle, S.Y.; Matee, M.I.; Langeland, N.; Mylvaganam, H. (2007). Identification of diarrheagenic Escherichia coli isolated from infants and children in Dar es Salaam, Tanzania. BMC Infect Dis. 9(7), 92-98.

11. NCCLS. (2005). Performance standards for antimicrobial disk susceptibility tests M2-A6. Wayne, Pennsylvania

12. Ozerol, I.H.; Bayraktar, M.R.; Iseri, L.; Otlu, B.; Durmaz, R. (2005) The prevalence and molecular typing of enterotoxigenic Escherichia coli strains isolated from diarrheic stools in Malatya, Turkey. New Microbiol. 28, 37-43. 
13. Qadri, F.; Das, S.K.; Faruque, A.S.; Fuchs, G.J.; Albert, M.J.; Sack, R.B.; Svennerholm, A.M. (2000). Prevalence of toxin types and colonization factors in enterotoxigenic Escherichia coli isolated during a 2-year period from diarrheal patients in Bangladesh. J Clin Microbiol. 38, 27-31.

14. Qadri, F.; Svennerholm, A.M.; Faruque, A.S.; Sack, R.B. (2005). Enterotoxigenic Escherichia coli in developing countries: epidemiology, microbiology, clinical features, treatment, and prevention. Clin Microbiol Rev. 18(3), 465-83

15. Shaheen, H.I.; Abdel Messih, I.A.; Klena, J.D.; Mansour, A.; El-
Wakkeel, Z.; Wierzba, T.F.; Sanders, J.W.; Khalil, S.B.; Rockabrand, D.M.; Monteville, M.R.; Rozmajzl, P.J.; Svennerholm, A.M.; Frenck, R.W. (2009). Phenotypic and genotypic analysis of enterotoxigenic Escherichia coli in samples obtained from Egyptian children presenting to referral hospitals. J. Clin. Microbiol. 47(1), 189-197.

16. Usein, C.R.; Tatu-Chitoiu, D.; Ciontea, S.; Condei, M.; Damian, M. (2009). Escherichia coli pathotypes associated with diarrhea in Romanian children younger than 5 years of age. Jpn J Infect Dis. 62(4), 289-293. 